\title{
Entwicklung von piezoresistiven Hydrogelsensoren zum Nachweis von Ethanol
}

\author{
Jan Erfkamp ${ }^{1}$, Margarita Günther ${ }^{1}$, Gerald Gerlach ${ }^{1}$ \\ ${ }^{1}$ Institut für Festkörperelektronik, Technische Universität Dresden, Deutschland \\ jan.erfkamp@tu-dresden.de
}

\section{Zusammenfassung}

Der Beitrag beschreibt einen kostengünstigen Sensor zur Detektion von Ethanol, z.B. in Alkoholika, der alkoholsensitive Hydrogele aus Acrylamid und Bisacrylamid mit piezoresistiven Sensoren kombiniert. Dabei wird die reversible Quellung bzw. Entquellung des Hydrogels gezeigt, welche Voraussetzung für einen reproduzierbaren Betrieb des Sensors ist. Weitere Faktoren wie der Einfluss der Acrylamid- und Bisacrylamid-Konzentration auf die Sensitivität und die mechanische Stabilität des Hydrogels sowie die Selektivität des Hydrogels gegenüber weiteren Alkoholen werden dargelegt. Erste Messungen in piezoresistiven Sensoren zeigen das große Anwendungspotenzial für die Detektion von Alkohol.

Keywords: Hydrogel,piezoresistiver Sensor,Ethanol,Alkohol,Prozessanalytik

\section{Motivation und Einleitung}

Im Brauereiwesen ist die EthanolKonzentration einer der essenziellen Parameter der Prozessanalytik. Standardmäßig eingesetzte Methoden wie Hochleistungsflüssigchromatographie (HPLC) [1] oder die enzymatische Alkoholbestimmung mittels UV-VIS-Spektroskopie [2] sind gerade für Kleinbrauereien enorm kostspielig und aufwendig. Alternative Methoden, wie z.B. Aräometer, bieten im Vergleich dazu eine nur unzureichende Genauigkeit. In diesem Beitrag wird deshalb ein piezoresistiver Siliziumsensor vorgestellt, der das ethanolabhängige Quellen von Hydrogelen ausnutzt und der ein großes Potenzial für eine preiswerte Herstellung, Miniaturisierbarkeit sowie InlineProzessfähigkeit besitzt.

Hydrogele sind "intelligente“ hydrophile Polymernetzwerke, die in Abhängigkeit von verschiedenen Stimuli, wie pH-Wert, der Salzkonzentration oder Temperatur, unterschiedliche Quelleigenschaften zeigen [3]. In Kombination mit piezoresistiven Drucksensoren, die den resultierenden Quelldruck messen, können so neuartige Sensoren für die Bio-, Umwelt- und Prozessanalytik entwickelt werden $[4,5,6]$.
Ein ethanolabhängiges Quellen wurde bereits für Hydrogele auf Basis von Poly( $N$-lsopropylacrylamid) [7,8] und Polyacrylsäure [9] gezeigt. In dieser Arbeit wird auf Basis von Polyacrylamid-Bisacrylamid-Hydrogelen ein piezoresistiver Hydrogelsensor entwickelt, welcher kostengünstig und mit hoher Sensitivität Ethanol, z.B. in Alkoholika, detektieren kann. Die Alkohol-Konzentration führt dabei zu einer reversiblen Quellung bzw. Entquellung des Hydrogels. Der Quelldruck auf der Biegeplatte des verwendeten Drucksensors führt zu einer piezoresistiven Widerstandsänderung und über eine Wheatstonesche Brückenschaltung zu einer Änderung des Messsignales, die proportional abhängig von der Ethanol-Konzentration ist.

\section{Material und Methoden}

Synthese von Polyacrylamid-BisacrylamidHydrogelen

Für die Synthese der Hydrogele wurden 10 Vol.-\% Acrylamid (Aam, Sigma Aldrich) und $0,44 \mathrm{~mol} \%$ Bisacrylamid (Bis, Carl Roth) in destilliertem Wasser gelöst und für 5 min mit Stickstoff entgast. Bei den Synthesen mit unterschiedlichen Acrylamid- und Bisacrylamid-Konzentrationen wurden die Verhältnisse entsprechend variiert. Im Anschluss wurde durch Zugabe von 0,05 Vol.-\% Ammonium- 
peroxodisulfat (Sigma Aldrich) und 0,5 Vol.-\% $N, N, N^{*}, N^{*}$-Tetramethylethylendiamin (Carl Roth) die Polymerisation initiiert. Das Gesamtvolumen eines Syntheseansatzes betrug $200 \mu \mathrm{l}$. Je $30 \mu \mathrm{l}$ eines Ansatzes wurden in eine Kunststoff-Waferform (5,32 mm x 5,32 mm x $0,76 \mathrm{~mm}$, Fluoroware) gegeben und polymerisierten dort für mindestens $6 \mathrm{~h}$. Um nicht umgesetzte Monomere aus den synthetisierten Polymeren zu entfernen, wurden die Hydrogele drei Tage in destilliertem Wasser gewaschen.

\section{Quellungsuntersuchungen in Alkohol-Wasser- Gemischen}

Nach der Synthese erfolgte zunächst eine fünfmalige Konditionierung der Hydrogele in entsprechender Alkohollösung. Im Anschluss wurden die Hydrogele in destilliertem Wasser gewaschen und nach $24 \mathrm{~h}$ mittels Mikroskopie die Ausgangslänge $I_{0}$ des Hydrogels bestimmt. Nach der Herstellung eines Ethanol-WasserGemisches mit entsprechendem Volumenanteil an Ethanol konnten nun in dieser Lösung die Hydrogele für $24 \mathrm{~h}$ inkubiert werden. Im Anschluss erfolgte die Bestimmung der Länge des Hydrogels I mittels Mikroskopie und die Lösungen konnten nun erneut gewechselt werden. Aus den Ergebnissen konnte nun der Quellungsgrad des Hydrogels $Q$ als relative Längenänderung [10] bestimmt werden (1):

$Q=\frac{l-l_{0}}{l_{0}} \times 100 \%$

\section{Sensorkonstruktion}

Für Applikationen im Sensor mussten zunächst definierte Hydrogel-Schichten hergestellt werden. Dazu wurde ein Kunststoff-Spacer (Dicke $=250 \mu \mathrm{m})$ zwischen zwei Objektträger gespannt und die Pregel-Lösung in die daraus resultierende Kavität (Volumen $250 \mu \mathrm{l}$ ) gefüllt. Nach der Polymerisation wurde das Gel drei Tage lang in destilliertem Wasser gewaschen. Nun wurden kreisförmige Hydrogele mit einem Durchmesser von $1,5 \mathrm{~mm}$ ausgestanzt und auf einen Silicium-Spacer (Dicke $280 \mu \mathrm{m}$ ) aufgebracht, ehe die Kavität des Drucksensors (EPCOS, Modell: C41, $5,0 \mathrm{~mm} \times 5,0 \mathrm{~mm} \times 0,4 \mathrm{~mm}$ ) mit dem Hydrogel bestückt und der Sensor mittels Cyanacrylat auf eine Leiterplatte fixiert wurde. Nach dem Bonden des Sensorchips an die Leiterplatte wurden Verbindungskabel zur Ausleseeinheit (Fluke 45 Multimeter) angelötet. Um die Ethanol-Wasser-Gemische zum Hydrogelsensor zuzuführen und zu analysieren, wurde je ein Zu- und Ablaufschlauch mittels Cyanacrylat und Zwei-KomponentenEpoxidharz auf der Leiterplatte fixiert. Der resultierende Sensor (Abb. 1) wurde an eine Schlauchpumpe mit einem konstanten Fluss von $0,2 \mathrm{ml} / \mathrm{min}$ angeschlossen. Für die Sensormessungen wurden nach jeweils $30 \mathrm{~min}$ die Ethanol-Wasser-Gemische gewechselt.

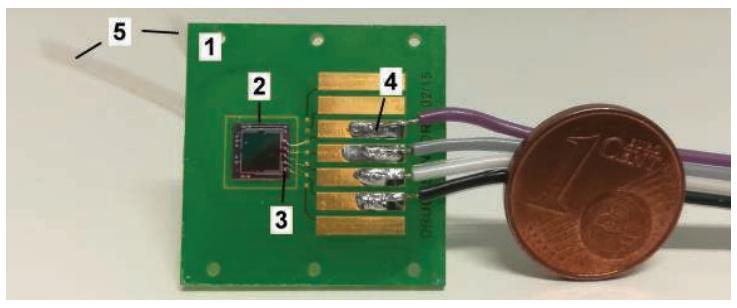

Abb. 1: Hydrogel-basierter Drucksensor zur Detektion von Ethanol; 1 Leiterplatte, 2 Drucksensorchip mit Hydrogel in der Kavität, 3 Bondinseln mit gebondeten Golddrähten, 4 gelötete Anschlusskabel, $5 \mathrm{Zu}$ - und Ablaufschläuche zur Pumpe.

\section{Ergebnisse und Diskussion \\ Reversibilität der Quellung}

Die Reproduzierbarkeit des Alkoholsensors ist entscheidend für Anwendungen in der Prozessanalytik. Hydrogele eignen sich durch ihren reversiblen Quell- und Entquellvorgang daher hervorragend für Anwendungen in der Sensorik (Abb. 2).
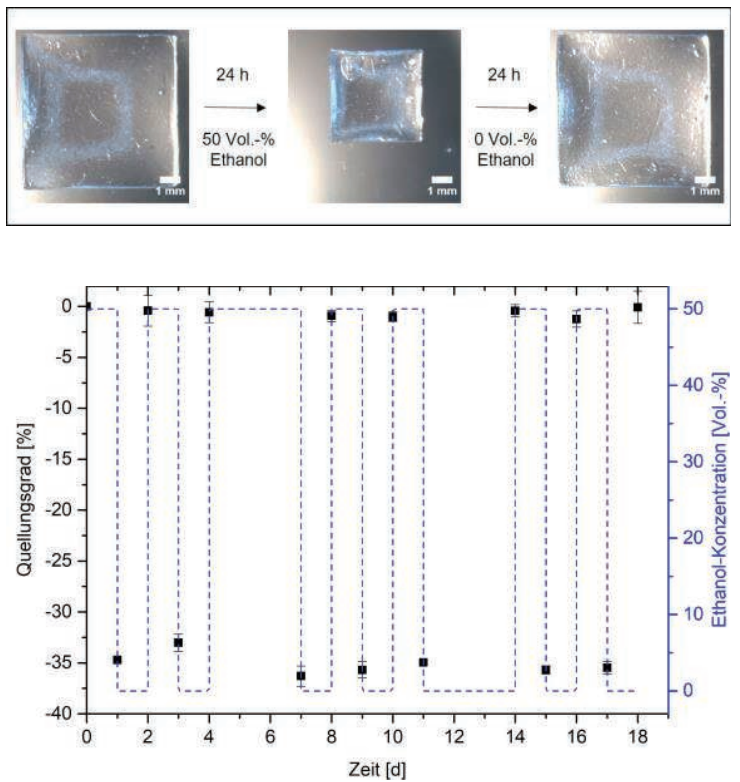

Abb. 2: Reversible Hydrogel-Quellung in Abhängigkeit von der EthanolKonzentration (Hydrogel: $10 \mathrm{Vol}-\mathrm{-} \%$ Aam und 0,44 mol\% Bis). 
Beim Wechsel von 0 Vol.-\% auf 50 Vol.- $\%$ Ethanol entquillt das Hydrogel um ca. $35 \%$. Bei einem erneuten Wechsel auf 0 Vol.-\% Ethanol nehmen die Hydrogele wieder ihre Ausgangsgrößen an. Dieses Verhalten konnte auch bei anschließenden Lösungsmittelwechseln reproduziert werden. Stimuliresponsive Hydrogele sind für die Reversibilität der Entquellung bereits bekannt, wie beispielweise an Temperatur- [11], pH- [12] und Salz-sensitiven [13] Hydrogelen gezeigt werden konnte.

Sensitivität des Hydrogels gegenüber unterschiedlichen Alkoholen

Für Anwendungen in der Alkoholdetektion sollte die Sensitivität in einem Bereich von $0-50$ Vol.-\% möglichst linear sein. Aus diesem Grunde wurde der Einfluss der AlkoholKonzentration auf das Quellverhalten der Hydrogele untersucht (Abb. 3).

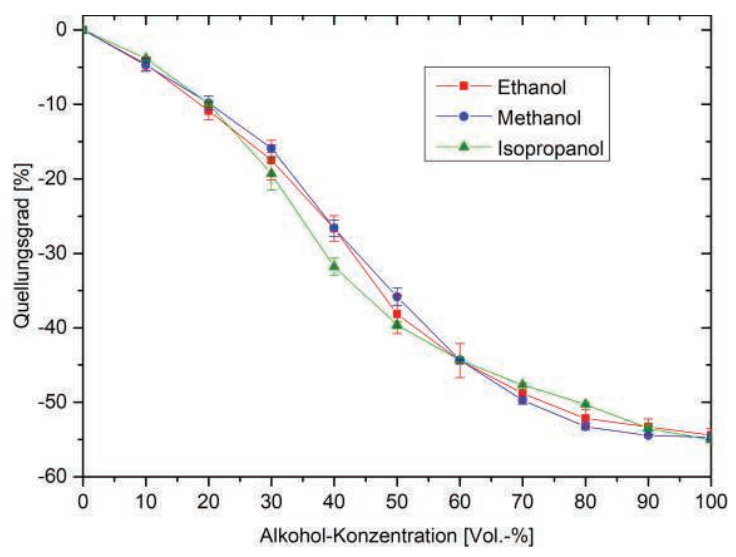

Abb. 3: Quellungsgrad der Hydrogele bei unterschiedlichen Alkohol-Konzentrationen von Ethanol, Methanol und Isopropanol (Hydrogel: $10 \mathrm{Vol}-\%$ Aam und 0,44 mol\% Bis).

Die Hydrogele zeigen neben der Ethanolabhängigkeit ebenfalls Sensitivitäten gegenüber weiteren Alkoholen wie Methanol und Isopropanol. Die Quersensitivität stellt für Anwendungen in der Alkoholanalytik jedoch kein Problem dar, da Alkoholika ausschließlich Ethanol enthalten. Alle Alkohole zeigen über den gesamten Konzentrationsverlauf ähnliche Sensitivitäten. Selbst bei geringen Alkoholkonzentrationen sind bereits Quellvorgänge detektierbar. Dies deutet auf eine geringe Nachweisgrenze hin, die insbesondere für die Alkoholdetektion im Brauereiwesen interessant sein könnte. Der Verlauf ist über einen großen Konzentrationsbereich quasi-linear. Im
Vergleich dazu entquellen Poly( $N$-Isopropylacrylamid)-Hydrogele in einem Bereich von $0-20$ Vol.-\%, von $20-40$ Vol.-\% ist nahezu keine Änderung des Quellungsgrades zu beobachten, ehe die Hydrogele in einem Bereich von 40-100 Vol.-\% wieder quellen [14]. Dieses nichtlineare Quellverhalten ist im Vergleich zum quasi-linearen Verhalten der Polyacrylamid-Hydrogele für sensorische Anwendungen jedoch ungünstig.

Einfluss der Acrylamid- und BisacrylamidKonzentration auf die Sensitivität

Die Zusammensetzung des Hydrogels beeinflusst das Quellungsverhalten und damit auch die Sensitivität des Sensors. Zunächst wurde der Einfluss der AcrylamidVolumenkonzentration auf das Quellungsverhalten der Hydrogele bestimmt (Abb. 4).

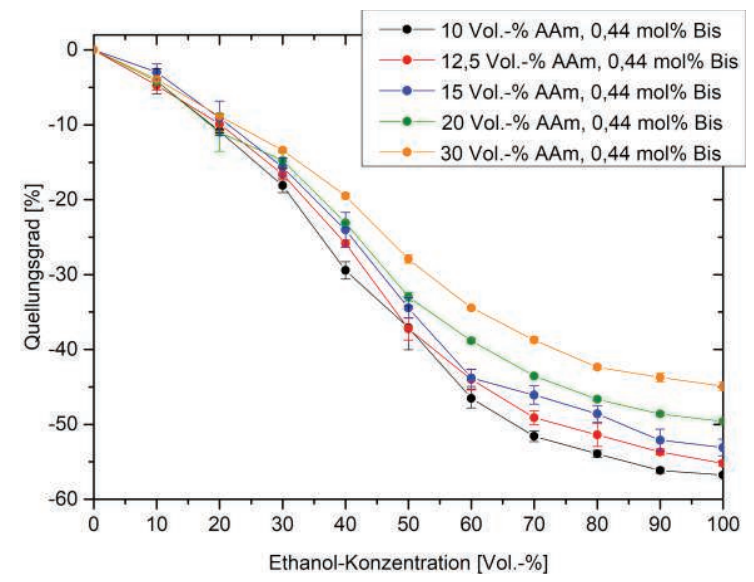

Abb. 4: Einfluss der Acrylamid-Konzentration auf den Quellungsgrad der Hydrogele.

Mit steigender Acrylamid-Konzentration nimmt die Sensitivität des Hydrogels ab. Die Zunahme der Kettenlänge des Polymers führt zu Überlappungen und Verschlaufungen von Polymerketten innerhalb des Hydrogels. Die resultierende physikalische Vernetzung verringert den Quellungsgrad [15], erhöht jedoch auch die mechanische Stabilität des Hydrogels. Geringere Konzentrationen an Acrylamid ( $\leq 7,5$ Vol.-\% AAm) führen zu einer Verschlechterung der mechanischen Eigenschaften, sodass diese Gele nicht mehr für sensorische Zwecke genutzt werden können.

Neben dem Monomer Acrylamid beeinflusst auch der Vernetzer Bisacrylamid das Quellungsverhalten (Abb. 5). 


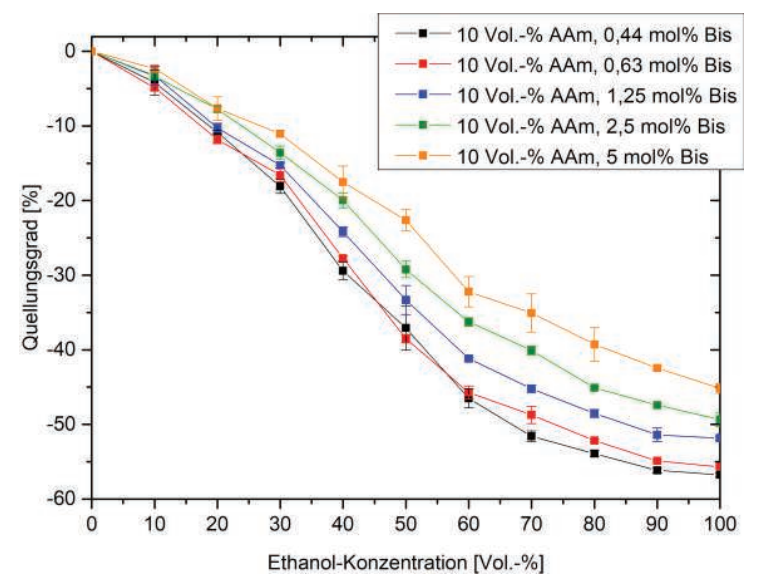

Abb. 5: Einfluss der BisacrylamidKonzentration auf den Quellungsgrad der Hydrogele.

Je geringer die Konzentration des Vernetzers Bisacrylamid ist, desto größer ist der Quellungsgrad der Hydrogele. Ein geringer Vernetzeranteil führt zu weniger Vernetzungspunkten und somit zu einer größeren Maschenweite im Hydrogel [15]. Dies führt zu einer höheren Sensitivität, jedoch auch zu einer Verringerung der mechanischen Stabilität des Gels. Bei geringeren Konzentrationen an Bisacrylamid ( $\leq 0,25 \mathrm{~mol} \%$ Bis) wird das Hydrogel mechanisch so instabil, dass es nicht mehr für die Anwendung nutzbar ist. Für Sensorapplikationen müssen die Hydrogele daher so variiert werden, dass eine möglichst hohe Sensitivität bei gleichzeitiger mechanischer Stabilität vorliegt.

\section{Piezoresistiver Ethanolsensor}

Die ethanolbedingte Quellung des Hydrogels verursacht ausreichend große Kräfte, um die Biegeplatte des Drucksensors zu deformieren, wodurch eine Ausgangsspannung $U_{\text {out }}$ messbar wird (Abb. 6).

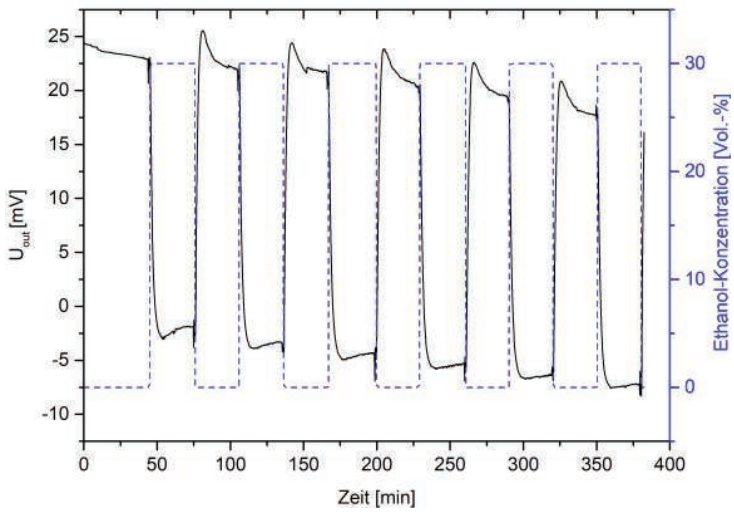

Abb. 6: Messung des Spannungsverlaufs eines piezoresistiven Hydrogelbasierten Drucksensors in Abhängigkeit von zyklischen Wechseln der Ethanol-Konzentration (Hydrogel: 10 Vol.-\% Aam und 0,44 mol\% Bis).

Mit der Messung kann im Sensor ebenfalls die Reproduzierbarkeit der Quellung des Hydrogels über mehrere Quellzyklen nachgewiesen werden. Dabei traten beim Wechsel von 0 Vol.- $\%$ auf $30 \mathrm{Vol} .-\%$ Signaländerungen von ca. $25 \mathrm{mV}$ auf. Aufgrund der großen Signaländerung ist zu erwarten, dass auch geringe Ethanolkonzentrationen wie im Bier ( 5 Vol.- $\%$ Ethanol) detektiert werden können. Diese Eigenschaft, ebenso wie die geringe Ansprechzeit des Sensors von wenigen Minuten und die kostengünstige Herstellung machen dieses Sensorkonzept besonders interessant für industrielle Anwendungen.

\section{Zusammenfassung}

In diesem Beitrag wurde die Eignung von Acrylamid-Bisacrylamid-Hydrogelen für die Detektion von Ethanol in wässrigen Lösungen gezeigt. Der Quelldruck des Hydrogels wurde dabei durch piezoresistive Drucksensoren gemessen. Es konnte gezeigt werden, dass Hydrogele mit 10 Vol.-\% Aam und 0,44 mol\% Bis zu ausreichend großen Sensitivitäten führten, sodass solche Sensoren in situ bei der Herstellung alkoholischer Getränke wie Bier oder hochprozentige Alkoholika eingesetzt werden können. Die Zusammensetzung hat dabei einen Einfluss auf die Sensitivität des Hydrogels. Das Hydrogel zeigt eine ähnliche Sensitivität auch gegenüber Methanol und Isopropanol. Die Sensitivität der Sensoren zeigt in einem großen Konzentrationsbereich einen sehr linearen Verlauf, was für den Einsatz als Ethanolsensoren in der Alkoholherstellung ein großer Vorteil ist. 


\section{Literaturverzeichnis}

[1] M. Castellari, E. Sartini, U. Spinabelli, C. Riponi, S. Galassi, Journal of Chromatographic Science 39, 235-238 (2001), doi:

10.1093/chromsci/39.6.235

[2] C. Korb, Entwicklung enzymatischer Screeningverfahren, Disseration, Martin-LutherUniversität Halle-Wittenberg (2003).

[3] M. Günther, T. Wallmersperger, G. Gerlach, Chemosensors 2, 145-170 (2014), doi: 10.3390/chemosensors2020145.

[4] M. Günther, Anwendung polymerer Funktionsschichten in piezoresistiven chemischen und Feuchtesensoren, Dresdner Beiträge zur Sensorik (Hrsg.: G. Gerlach), Band 38, TUDpress, Dresden (2009).

[5] M. Günther, G. Gerlach, Hydrogels for chemical sensors in Hydrogel Sensors and Actuators Engineering and Technology (Hrsg.: G. Gerlach, K.-F. Arndt), Springer Berlin Heidelberg, S. 165-195 (2009).

[6] U. Schmidt, C. Jorsch, M. Günther, G. Gerlach, Journal of Sensors and Sensor Systems 5, 409-417 (2016), doi: 10.5194/jsss-5-409-2016.

[7] K. Mukae, M. Sakurai, S. Sawamura, K. Makino, S. W. Kim, I. Ueda, K. Shirahama, The Journal of Physical Chemistry 97, 131-141 (1993), doi: 10.1021/j100105a034.

[8] K.-F. Arndt, D. Kuckling, A. Richter, Polymers for Advanced Technologies 11, 496-505 (2000), doi: 10.1002/1099-1581(200008/12) 11:8/12<496::AID-PAT996>3.0.CO;2-7.

[9] Y. Nishiyama, M. Satoh, Macromolecular Rapid Communications 21, 174-177 (2000), doi: 10.1002/(SICI)1521-3927(200003)21:4<174:: AID-MARC174>3.0.CO;2-K.

[10] W. Bai, N. A. Gariano, D. A. Spivak, Journal of the American Chemical Society 135, 6977-6984 (2013), doi: 10.1021/ja400576p.

[11] M. Guenther, G. Gerlach, D. Kuckling, K. Kretschmer, C. Corten, J. Weber, J. Sorber, G. Suchaneck, K.-F. Arndt in Smart structures and materials 2006: smart sensor monitoring systems and applications (Hrsg.: D. Inaudi, W. Ecke, B. Culshaw, K. J. Peters, E. Udd), Proc SPIE, 6167, San Diego.

[12] X. J. Liu, H. Q. Li, B. Y. Zhang, Y. J. Wang, X. Y. Ren, S. Guan, G. H. Gao, RSC Advances 6, 4850-4857 (2016), doi: 10.1039/c5ra24414e.

[13] H. Izawa, Y. Kaneko, J. Kadokawa, Journal of Materials Chemistry 19, 6969-6972 (2009), doi: 10.1039/b916864h

[14] A. Richter, Quellfähige Polymernetzwerke als Aktor-Sensor-Systeme für die Fluidtechnik, Disseration, Technische Universität Dresden (2002).

[15] H. Wack, Zum Quellungsdruck von polymeren Hydrogelen, Universität Duisburg-Essen (2006).

\section{Danksagung}

Die Autoren danken der Deutschen Forschungsgemeinschaft (DFG) für die finanzielle Unterstützung im Rahmen des Graduiertenkollegs „Hydrogel-basierte Mikrosysteme" (GRK 1865). 